Relativistic Multireference Many-body

Perturbation Theory for Open-shell lons with

Multiple Valence Shell Electrons: the Transition

Rates and Lifetimes of the Excited Levels in

Chlorinelike Fe X

Y. Ishikawa, J. A. Santana, E. Träbert

October 1, 2009

Journal of Physics B: Atomic Molecular and Optical Physics 
This document was prepared as an account of work sponsored by an agency of the United States government. Neither the United States government nor Lawrence Livermore National Security, LLC, nor any of their employees makes any warranty, expressed or implied, or assumes any legal liability or responsibility for the accuracy, completeness, or usefulness of any information, apparatus, product, or process disclosed, or represents that its use would not infringe privately owned rights. Reference herein to any specific commercial product, process, or service by trade name, trademark, manufacturer, or otherwise does not necessarily constitute or imply its endorsement, recommendation, or favoring by the United States government or Lawrence Livermore National Security, LLC. The views and opinions of authors expressed herein do not necessarily state or reflect those of the United States government or Lawrence Livermore National Security, LLC, and shall not be used for advertising or product endorsement purposes. 


\title{
Relativistic multireference many-body perturbation theory for open-shell ions with multiple valence shell electrons: the transition rates and lifetimes of the excited levels in chlorinelike Fe X
}

\author{
Yasuyuki Ishikawa ${ }^{1}$, Juan A Santana ${ }^{1}$ and Elmar Träbert ${ }^{2,3}$ \\ ${ }^{1}$ Department of Chemistry and the Chemical Physics Program, University of Puerto \\ Rico, P. O. Box 23346, San Juan, Puerto Rico 00931-3346, USA \\ ${ }^{2}$ Astronomisches Institut, Ruhr-Universität Bochum, D-44780 Bochum, Germany \\ ${ }^{3}$ Physics Division, LLNL, P.O. Box 808, Livermore, CA 94551, USA \\ E-mail: yishikawa@uprrp.edu
}

\begin{abstract}
A recently developed relativistic multireference many-body perturbation theory based on multireference configuration-interaction wavefunctions as zeroth order wavefunctions is outlined. The perturbation theory employs a general class of configuration-interaction wave functions as reference functions, and thus is applicable to multiple open valence shell systems with near degeneracy of a manifold of strongly interacting configurations. Multireference many-body perturbation calculations are reported for the ground and excited states of chlorinelike $\mathrm{Fe} \mathrm{X}$ in which the near degeneracy of a manifold of strongly interacting configurations mandates a multireference treatment. Term energies of a total of 83 excited levels arising from the $3 \mathrm{~s}^{2} 3 \mathrm{p}^{5}, 3 \mathrm{~s} 3 \mathrm{p}^{6}, 3 \mathrm{~s}^{2} 3 \mathrm{p}^{4} 3 \mathrm{~d}, 3 \mathrm{~s} 3 \mathrm{p}^{5} 3 \mathrm{~d}$, and $3 \mathrm{~s}^{2} 3 \mathrm{p}^{3} 3 \mathrm{~d}^{2}$ configurations of the ion are evaluated to high accuracy. Transition rates associated with E1/M1/E2/M2/E3 radiative decays and lifetimes of a number of excited levels are calculated and compared with laboratory measurements to critically evaluate recent experiments.
\end{abstract}

PACS numbers: $3270 \mathrm{Cs}, 3115 \mathrm{am}, 3450 \mathrm{Fa}$

Submitted to: J. Phys. B: At. Mol. Opt. Phys. Special Issue on "High Precision in Atomic Physics"

\section{Introduction}

Since important physical and chemical processes involve heavy atoms and highly-ionized ions, there has been increasing interest in the development of relativistic many-body theories $[1,2,3,4,5,6,7,8,9,10,11,12,13,14,15,16,17,18,19,20,21,22$, $23,24,25]$ for an accurate description of spectroscopic properties. Because relativistic and correlation effects are intertwined and play an essential role in the electronic structure and spectroscopic properties of many-electron systems, relativistic many-body 
perturbation theory (MBPT) and relativistic coupled cluster (CC) theory have become the subject of active research interest.

An important feature in the many-body algorithm is the highly correlated statespecific many-electron wavefunctions that accurately account for relativity and for nondynamic as well as dynamic correlation energy corrections arising from the effective electron-electron interaction - the instantaneous Coulomb and Breit interactions - in addition to the QED corrections.

During the past decades, most relativistic atomic structure calculations have been carried out by either finite-difference multiconfiguration (MC) Dirac-Fock selfconsistent field (DF SCF) [26, 27, 28], relativistic configuration interaction (CI) [9, 29], and relativistic many-body perturbation theory (MBPT) based on single-configuration DF SCF wave functions expanded in analytic basis sets [30, 31]. Each of these methods has strengths and weaknesses because their accuracy is restricted to different sectors of many-electron correlation. The inability of MC DF SCF and relativistic CI methods to make quantitative predictions in agreement with experiment [32] is well understood: These methods are most effective in treating nondynamic correlation (i.e. near-degeneracy in the valence shells), but fail to account for the bulk of dynamic correlation [21]. The single-reference MBPT has exactly the opposite characteristic; it is effective in accurately describing dynamic correlation, but fails to account for nondynamic correlation. Dynamic correlation is a short-range effect that arises from electron-electron interaction and is the major correction to the Dirac-Fock independent particle model, while nondynamic correlation is a consequence of the existence of nearly degenerate excited states that interact strongly with the reference state [11, 19, 21]. Near-degeneracy in the valence spinors gives rise to a manifold of strongly interacting configurations, i.e., strong configuration mixing within a relativistic complex [24], and makes a MC treatment mandatory. The classic examples in atomic physics are the near-degeneracy effects in ground-state alkaline-earth metals [17] and open-shell atoms with two or more valence-shell electrons [8, 19, 24, 25].

Once the near-degeneracy effects are accounted for by matrix MC DF SCF or relativistic CI along with the QED effects [33], the remaining dynamic correlation may be recovered by a relativistic multireference(MR)-MBPT [19, 21, 24], MR-CC [7], or MR-CI based on the MC reference functions [9, 34]. The relativistic MR-CI approach $[9,34]$, however, becomes quickly unwieldy in systems with large numbers of electrons, because the order of the MR-CI matrix increases rapidly as the number of electrons increases. Once the nondynamic correlation among valence electrons is treated, the remaining correlation may be recovered by second-order perturbation theory because it consists mainly of dynamic pair correlation due to short-range fluctuation potentials.

In recent studies [19, 21, 24], we have developed a relativistic MR-MBPT algorithm that combines the strengths of both relativistic CI and many-body perturbation methods and which yields highly accurate term energies for open-shell systems with multiple valence-shell electrons. The relativistic MR-MBPT, which underpins the electron correlation in strongly interacting many-electron systems, is the outstanding example 
of a successful many-body theory, one which predicts with high precision the outcome of spectroscopic experiments. The MR-MBPT perturbation calculations reported for the ground and low-lying odd- and even-parity excited states of Al-, Si-, and S-like ions $[19,21,24]$ have demonstrated unprecedented accuracy for systems with multiple valence electrons.

In the present study, we outline a procedure by which to perform the recently developed relativistic MR-MBPT theory [21, 24] in application to a general class of strongly correlated quasidegenerate systems. The essential feature of the theory is its underpinning of correlation corrections through treatment of the nondynamic correlation in zero order through quadratically convergent matrix multiconfiguration Dirac-FockBreit self-consistent field (MCDFB SCF) followed by relativistic CI [21, 24], and recovery of the remaining correlation, which is predominantly dynamic pair correlation, by second-order MR-MBPT theory. The relativistic MR-MBPT calculations reported in this study for the ground and low-lying odd- and even-parity excited states of chlorinelike iron demonstrate unprecedented accuracy for strongly correlated systems with multiple valence-shell electrons. Highly accurate term values for all the excited states arising from the $3 \mathrm{~s}^{2} 3 \mathrm{p}^{5}, 3 \mathrm{~s} 3 \mathrm{p}^{6}, 3 \mathrm{~s}^{2} 3 \mathrm{p}^{4} 3 \mathrm{~d}, 3 \mathrm{~s} 3 \mathrm{p}^{5} 3 \mathrm{~d}$, and $3 \mathrm{~s}^{2} 3 \mathrm{p}^{3} 3 \mathrm{~d}^{2}$ configurations, including the poorly determined levels arising from the $3 \mathrm{~s}^{2} 3 \mathrm{p}^{4} 3 \mathrm{~d}, 3 \mathrm{~s} 3 \mathrm{p}^{5} 3 \mathrm{~d}$, and $3 \mathrm{~s}^{2} 3 \mathrm{p}^{3} 3 \mathrm{~d}^{2}$ configurations, are reported. Theoretical E1/M1/E2/M2/E3 decay rates and lifetimes of the excited levels arising from the $3 \mathrm{~s}^{2} 3 \mathrm{p}^{5}, 3 \mathrm{~s} 3 \mathrm{p}^{6}, 3 \mathrm{~s}^{2} 3 \mathrm{p}^{4} 3 \mathrm{~d}$ configirations are computed to compare with and critically evaluate recent experiments.

\section{Relativistic MR-MBPT calculations}

The effective $N$-electron Hamiltonian for the development of our relativistic MRMBPT algorithm is taken to be the relativistic "no-pair" Dirac-Coulomb-Breit (DCB) Hamiltonian $H_{D C B}^{+}[35,36]$. The initial step in our MR-MBPT procedure is to determine a set of Dirac spinors via the MCDFB SCF for the subsequent many-body description of many-electron systems. Second-order variation of the state-averaged energy is taken with respect to the matrix elements of spinor unitary rotation matrix and configuration mixing coefficients in the MCDFB SCF wave function, leading to the Newton-Raphson equations for second-order MCDFB SCF [34]. This stateaveraged second-order MCDFB equation yields a well-balanced set of spinors suitable for describing the ground and low-lying even- and odd-parity excited levels [21].

For the chlorinelike ions, the state-averaged MCDFB SCF includes a total of 31 configuration-state functions (CSF) of even- and odd-parity $\pi$ with total angular momentum $\mathcal{J}=1 / 2-9 / 2,\left\{\Phi_{I}^{(+)}\left(\gamma_{I} \mathcal{J} \pi\right)\right\}\left(\in \mathfrak{P}_{M C D F}^{(+)}\right)$, arising from the $3 \mathrm{~s}^{2} 3 \mathrm{p}^{5}, 3 \mathrm{~s} 3 \mathrm{p}^{6}$, and $3 \mathrm{~s}^{2} 3 \mathrm{p}^{4} 3 \mathrm{~d}$ configurations, to determine a single set of spinors for the MR-CI and MRMBPT calculations that follow. The key to high-accuracy algorithm for multi-valenceelectron systems is the determination of a single set of spinors via the state-averaged MC DFB SCF, which provides a well-balanced description of the ground and excited levels. 
In order to account for nondynamic correlation, or strong configuration mixing among the quasi-degenerate open-shell states, the MR-CI for the ground and lowlying excited $\mathcal{J}=1 / 2-11 / 2$ states in the chlorinelike ions were subsequently carried out including a total of 3,097 (1,538 even- and 1,559 odd-parity) CSFs arising from the configurations $3 \mathrm{~s}^{m} 3 \mathrm{p}^{n} 3 \mathrm{~d}^{p}$, with $m+n+p=7$ and $p \leq 4$. Variation of the configurationstate coefficients $\left\{C_{I K}\right\}$ leads to the determinantal CI equation.

$$
\operatorname{det}\left(\left\langle\Phi_{I}^{(+)}\left(\gamma_{I} \mathcal{J} \pi\right)\left|H_{D C B}^{+}\right| \Phi_{J}^{(+)}\left(\gamma_{J} \mathcal{J} \pi\right)\right\rangle-E^{C I}\left\langle\Phi_{I}^{(+)}\left(\gamma_{I} \mathcal{J} \pi\right) \mid \Phi_{J}^{(+)}\left(\gamma_{J} \mathcal{J} \pi\right)\right\rangle\right)=0(1)
$$

The eigenfunctions $\left\{\psi_{K}^{C I}\left(\gamma_{K} \mathcal{J} \pi\right)\right\}$ form a CI subspace $\mathfrak{P}_{C I}^{(+)}$of the positive-energy space $\mathfrak{D}^{(+)}$,

$$
\psi_{K}^{C I}\left(\gamma_{K} \mathcal{J} \pi\right)=\sum_{I}^{M_{C I}} C_{I K} \Phi_{I}^{(+)}\left(\gamma_{I} \mathcal{J} \pi\right), K=1,2, . . M_{C I}\left(\in \mathfrak{P}_{C I}^{(+)}\right) .
$$

The eigenfunctions $\left\{\psi_{K}^{C I}\left(\gamma_{K} \mathcal{J} \pi\right)\right\}$ are multideterminantal wavefunctions, obtained by diagonalizing the DCB Hamiltonian within the CI subspace $\mathfrak{P}_{C I}^{(+)}$of the positive-energy space $\mathfrak{D}^{(+)}$in a given set of CSFs $\left\{\Phi_{I}^{(+)}\left(\gamma_{I} \mathcal{J} \pi\right)\right\}$.

The MR-CI accounts for the near degeneracy in energy levels, or nondynamic correlation, inherent in multi-valence-electron systems. The frequency-dependent Breit interaction $(\Delta B(\omega))$, normal mass shift (NMS) and specific mass shift (SMS) are evaluated at this stage as the first-order corrections using the eigenvectors $\left\{\psi_{K}^{C I}\left(\gamma_{K} \mathcal{J} \pi\right)\right\}$ from the MR-CI [21].

While the MR-CI accounts well for the near degeneracy in energy, it fails to accurately account for dynamic correlation. Therefore, each of the CI eigenstates is subjected to state-specific MR-MBPT refinement to account for the residual dynamic correlation to second-order of perturbation theory, using the multideterminantal CI eigenfunctions $\left\{\psi_{K}^{C I}\left(\gamma_{K} \mathcal{J} \pi\right)\right\}$ as the zero-order reference function.

$$
\begin{aligned}
& E_{K}^{(2)}=<\psi_{K}^{C I}\left(\gamma_{K} \mathcal{J} \pi\right)|V \mathcal{R} V| \psi_{K}^{C I}\left(\gamma_{K} \mathcal{J} \pi\right)> \\
& =\sum_{I, J=1}^{\mathfrak{P}_{C I}(+)} C_{I K} C_{J K}<\Phi_{I}^{(+)}\left(\gamma_{I} \mathcal{J} \pi\right)|V \mathcal{R} V| \Phi_{J}^{(+)}\left(\gamma_{J} \mathcal{J} \pi\right)>
\end{aligned}
$$

The $E_{K}^{(2)}$ represents the residual dynamic correlation - the dynamic correlation between the core and valence electrons unaccounted for in the MR-CI - to second order of perturbation theory. $\mathcal{R}$ is the resolvent operator,

$$
\mathcal{R}=\frac{\mathcal{Q}^{(+)}}{E_{K}^{C S F}-H_{0}} \text { with } \mathcal{Q}^{(+)}=\sum_{I}^{\mathcal{Q}(+)}\left|\Phi_{I}^{(+)}\left(\gamma_{I} \mathcal{J} \pi\right)><\Phi_{I}^{(+)}\left(\gamma_{I} \mathcal{J} \pi\right)\right| .
$$

The projection operator $\mathcal{Q}^{(+)}$projects onto the subspace $\mathfrak{Q}^{(+)}=\mathfrak{D}^{(+)}-\mathfrak{P}_{C I}^{(+)}$spanned by $\operatorname{CSFs}\left\{\Phi_{I}^{(+)}\left(\gamma_{I} \mathcal{J} \pi\right) ; I=M_{C I}+1, M_{C I}+2, \ldots\right\}$.

Each of the 3,097 CI eigenstates was subjected to state-specific MR-MBPT refinement to account for the residual dynamic correlation to second order of 
perturbation theory $[19,21,24]$. All electrons are included in the MR-MBPT perturbation theory calculations to determine accurately the valence-core correlation as well as the effects of relativity on electron correlation.

Radiative corrections, the Lamb shifts (LS), were estimated for each state by evaluating the electron self-energy and vacuum polarization following an approximation scheme discussed by Indelicato, Gorceix, and Desclaux [37]. The code described in Refs. [37] and [38] was adapted to our basis set expansion calculations for this purpose: All the necessary radial integrals were evaluated analytically. In this ratio method [38], the screening of the self energy is estimated by integrating the charge density of a spinor to a short distance from the origin, typically 0.3 Compton wavelength. The ratio of the integral computed with an MCDFB SCF spinor and that obtained from the corresponding hydrogenic spinor is used to scale the self-energy correction for a bare nuclear charge as computed by Mohr [39].

The large and small radial components of the Dirac spinors are expanded in sets of even-tempered Gaussian-type functions (GTF) [40, 41] that satisfy the boundary conditions associated with the finite nucleus [42, 43]. The speed of light is taken to be 137.0359895 a.u. throughout this study. The GTFs that satisfy the boundary conditions associated with the finite nucleus are automatically kinetically balanced [42]. Eventempered basis sets ( $\alpha=0.10$ and $\beta=2.0$ ) of $26 \mathrm{~s} 24 \mathrm{p} 20 \mathrm{~d}$ G spinors (G for "Gaussian") for up to angular momentum $L=2,18 \mathrm{G}$ spinors for $L=3$, and $15 \mathrm{G}$ spinors for $L=4$ 11 are employed. The order of the partial-wave expansion $L_{\max }$, the highest angular momentum of the spinors included in the virtual space, is $L_{\max }=11$ throughout this study. MR-MBPT correlation energy contributions to transition energies from partial wave $L=12$ and higher are on the order of $10 \mathrm{~cm}^{-1}$. The nuclei were simulated as spheres of uniform proton charge [42] with the radii $R(B o h r)=2.2677 \times 10^{-5} A^{1 / 3}$, where $A$ is the atomic mass (amu).

\section{Transition probabilities}

Recent relativistic many-body perturbation theory studies by Johnson, Plante, and Sapirstein [45] on heliumlike ions have laid the foundation for high accuracy calculations of reduced matrix elements and transition rates in one- and two-valence electron systems and demonstrated the capacity of theoretical methods to predict E1 and M1 transition rates accurately. In the present study, we have employed our first-order MR-MBPT wavefunctions to evaluate the transition rates in the Babushkin gauge, including the negative-energy space.

The first-order transition amplitude for high-accuracy calculations of transition rates is expressed in terms of CSFs,

$$
<T_{J}^{\vartheta}>_{K K^{\prime}}^{(1)}=\sum_{L=M+1}^{\mathfrak{Q}(+,-)} \sum_{I, I^{\prime}=1}^{\mathfrak{P}(+)} C_{I K} C_{I^{\prime} K^{\prime}}^{\prime}\left[\frac{<\Phi_{I}^{(+)}|V| \Phi_{L}^{(+,-)}><\Phi_{L}^{(+,-)}\left|T_{J M}^{\vartheta}\right| \Phi_{I^{\prime}}^{(+)}>}{E_{I}^{C S F}-E_{L}^{C S F}}\right.
$$




$$
\left.+\frac{<\Phi_{I}^{(+)}\left|T_{J M}^{\vartheta}\right| \Phi_{L}^{(+,-)}><\Phi_{L}^{(+,-)}|V| \Phi_{I^{\prime}}^{\prime(+)}>}{E_{I^{\prime}}^{C S F}-E_{L}^{C S F}}\right] .
$$

Summation $L$ over intermediate states $\Phi_{L}^{(+,-)}$includes both the positive $(\mathfrak{Q}(+))$ and negative $(\mathfrak{Q}(-))$ energy subspaces [45]. With the summation extended to negative energy subspace, E1 and E2 transition probabilities computed in the Coulomb gauge approaches the value computed in the Babushkin gauge. The corrections arising from approximate photon frequency may be eliminated semiempirically using experimental transition energies. In the present study, transition energies (and photon frequencies $\left.\omega^{(0+1+2)}\right)$ calculated by MR-MBPT second-order perturbation theory are close to the experimental values, and the terms arising from corrections to the photon frequency $\delta \omega=\omega^{\exp t}-\omega^{(0+1+2)}$ in both zero- and first-order transition amplitudes are significantly smaller and may be neglected.

Because of strong coupling between the large and small components of the Dirac 4-spinors in the transition matrix elements, particularly E2 transition probabilities evaluated by excluding the negative-energy space in the Coulomb gauge are inaccurate and deviate from the values evaluated in the Babushkin gauge. When contributions from the negative energy space are included (Eq. 5), transition probabilities evaluated in Coulomb gauge approach those evaluated in Babushkin gauge.

\section{Results and discussion}

\subsection{Term energies of $n=3$ levels}

Theoretical term energies of the excited states arising from the $3 \mathrm{~s}^{2} 3 \mathrm{p}^{5}, 3 \mathrm{~s} 3 \mathrm{p}^{6}, 3 \mathrm{~s}^{2} 3 \mathrm{p}^{4} 3 \mathrm{~d}$, $3 \mathrm{~s} 3 \mathrm{p}^{5} 3 \mathrm{~d}$, and $3 \mathrm{~s}^{2} 3 \mathrm{p}^{3} 3 \mathrm{~d}^{2}$ configurations are compared with the hitherto available experimental data in Table 1. Experimental term energies reproduced are those compiled in the NIST Atomic Spectra Database [46] and the assessed sets of atomic data by Del Zanna et al. [47]. None of the excited levels arising from the $3 \mathrm{~s}^{2} 3 \mathrm{p}^{3} 3 \mathrm{~d}^{2}$ configuration has been experimentally identified.

Of the computed 83 excited levels in Fe X, experimental term energies are available only for 30 low-lying levels in the NIST database. For these levels, the theoretical term energies are in excellent agreement with NIST data. Theory-experiment deviations are at the $0.01 \%$ level for all but eight levels, where the deviations range from 0.11 to 1.15 $\%$. For the $3 \mathrm{~s}^{2} 3 \mathrm{p}^{4} 3 \mathrm{~d}^{2} \mathrm{P}_{3 / 2}$ and ${ }^{2} \mathrm{~F}_{5 / 2}$ levels in particular, the deviations are significant, in the $0.29-1.15 \%$ range. We argue that these experimental term energies adopted in the NIST database are based on misidentified lines, resulting in the large theoryexperiment deviation. To resolve the discrepancies for these levels, more accurate line identifications are needed. In a recent study [47], Del Zanna et al. have collected atomic data from different sources and assessed them to provide term energies of the 54 levels reproduced in Table 1. Many of these levels benchmarked by Del Zanna et al. agree to the $0.01 \%$ level with theory, although 20 excited levels arising from the $3 \mathrm{~s}^{2} 3 \mathrm{p}^{4} 3 \mathrm{~d}$, $3 \mathrm{~s} 3 \mathrm{p}^{5} 3 \mathrm{~d}$ and $3 \mathrm{~s}^{2} 3 \mathrm{p}^{3} 3 \mathrm{~d}^{2}$ configurations exhibit significant deviations, ranging from 0.32 
$\%$ to $1.35 \%$. The term energies computed by Aggarwal and Keenan [48, 49] using a relativistic CI method are reproduced in the last column of the table. Whereas the CI method accounts well for the nondynamic correlation among the valence electrons in the $\mathrm{n}=3$ shells, it fails to account for the bulk of dynamic correlation between the core and valence electrons. Therefore, the CI-calculated term energies deviate from experiment by up to ca. $3 \%$. In contrast, the MR-MBPT-calculated term energies are expected to be accurate to the $0.01 \%$ level.

\subsection{Transition rates and lifetimes}

Transition rates in atoms and ions reflect atomic structure and dynamics in ways that are related to the level structure, but that also depend differently on atomic parameters. The electric dipole (E1) operator features a dependence on the notoriously tricky radial wave functions that is not tested by levels optimized under the Ritz variation principle. Spinchanging (intercombination) E1 transitions depend on relativity and on electron-electron magnetic interactions of which the Breit operator is merely an approximation, and E1forbidden transitions are sensitive to details of the wave function composition. Cl-like ions feature examples of all these cases, and since very different orders of magnitude of level lifetimes (the inverse of the sum of all decay rates from a given level) are involved, experiments on Cl-like ions have employed beam-foil spectroscopy (see references in $[50,51]$, synchrotron light excitation of noble gases [52], stored ion beams [53, 54, 55, 56], electrostatic ion traps [57, 58,59,60], and electron beam ion traps [61, 62]. Not all of these techniques are applicable to Fe X (yet). In the following, we discuss examples and compare their results for Fe $\mathrm{X}$ to the results of our calculations. The selection of our examples is guided by longevity; that means, we do not discuss levels that readily decay as in a hydrogenlike ion, but only those E1 decays that connect the lowest displaced term $\left(3 \mathrm{~s} 3 \mathrm{p}^{6}{ }^{2} \mathrm{~S}\right)$ to the $3 \mathrm{~s}^{2} 3 \mathrm{p}^{5}{ }^{2} \mathrm{P}^{\mathrm{o}}$ ground term, the E1-forbidden transition within the ground term, and intercombination or E1-forbidden decays of $3 \mathrm{~s}^{2} 3 \mathrm{p}^{4} 3 \mathrm{~d}$ levels. For a level scheme, see figure 1. Table 2 displays theoretical E1/M1/E2/M2/E3 decay rates and lifetimes of the excited levels arising from the $3 \mathrm{~s}^{2} 3 \mathrm{p}^{5}, 3 \mathrm{~s} 3 \mathrm{p}^{6}$, and $3 \mathrm{~s}^{2} 3 \mathrm{p}^{4} 3 \mathrm{~d}$ configurations in Fe X.

For the lowest excited level, $3 \mathrm{~s} 3 \mathrm{p}^{6}{ }^{2} \mathrm{~S}$, there is good agreement of our calculations with both the level lifetime and the branch fraction of the decays with the results of the only (beam-foil) data set available [50]. Quite a number of calculations have addressed this line doublet in the $\mathrm{Cl}$ isoelectronic sequence (for references, see $[50,51,63,64,65]$ ). The largest differences are evident at the low end of the sequence, which is outside of the scope of this report.

Within the ground term, a magnetic dipole transition (with a very weak, less than $0.1 \%$, electric quadrupole admixture) connects the $J=1 / 2$ and $J=3 / 2$ fine structure levels. The corresponding solar corona line has been dubbed the "red iron line'. Because of the fundamental interest in such prominent solar coronal features, the transition rate has frequently been calculated (see time line in figure 2), but 


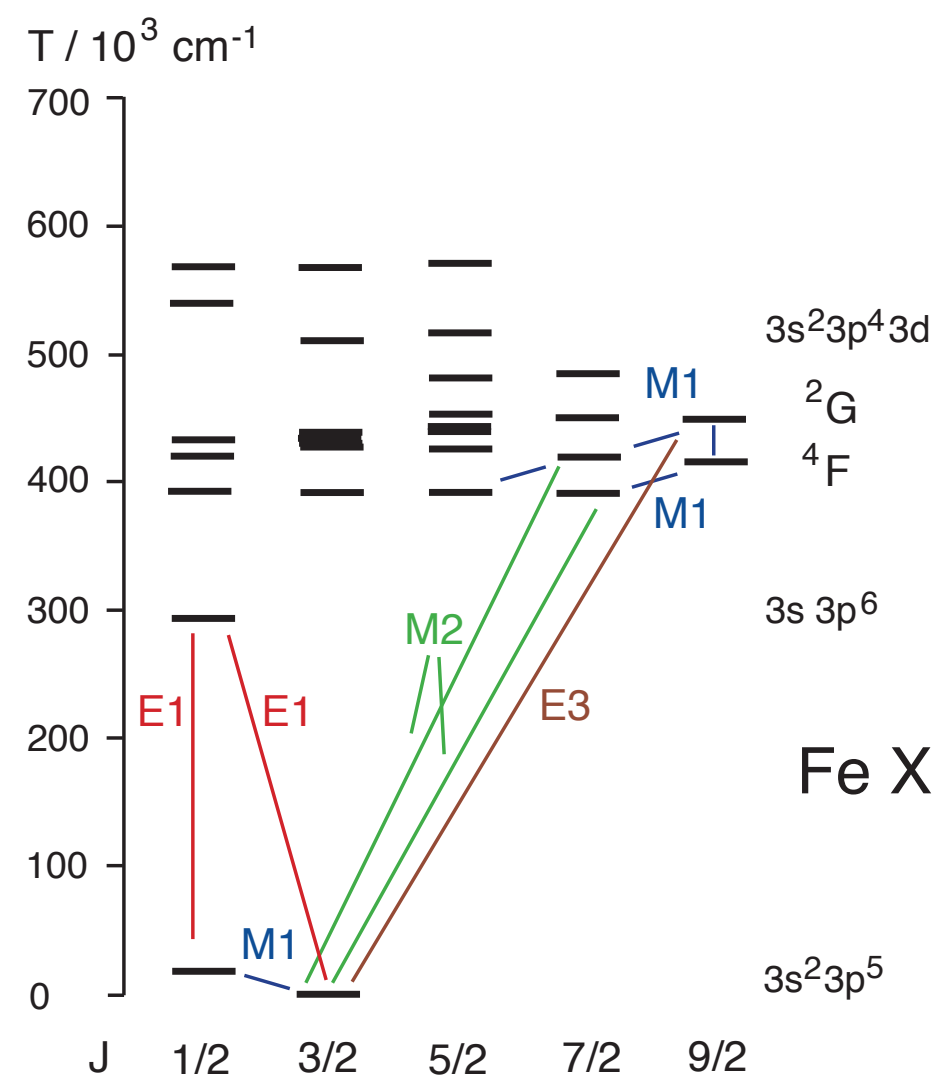

Figure 1. (Colour online) Lowest levels of Fe X and dominant decays of long-lived levels listed in table 2.

most of the early calculations have failed to reproduce the experimental fine structure splitting (known from the wavelength), and whatever energy splitting calculated was then 'semiempirically adjusted' by replacing it with the experimental data. The the results of such a procedure all lie close to each other, but are of little intrinsic merit beyond pointing out in which range the correct value should be expected. In contrast, the results of the few early $a b$ initio calculations scatter notably from the expected range of results, mostly reflecting the shortcomings of the fine structure interval calculation. The corresponding transition rate in Fe XIV (the 'green iron line') has been measured with utmost precision [66, 67], but the experimental situation is much less suitable for precise measurements in Fe X, because of the roughly $630 \mathrm{~nm}$ wavelength which is in a range where typical bialkali photomultipliers have a poor quantum efficiency and thus a poor signal-to-noise ratio. The early result of experiments with an electrostatic ion trap $[57,58,59]$ deviated well beyond the stated error bar from the expected lifetime value range. Measurements at a heavy-ion storage ring were tried [53] were tried, but failed to see the transition; instead similar experiments were then conducted on the isoelectronic spectra of $\mathrm{Co}, \mathrm{Ni}$, and $\mathrm{Cu}[54,55,56]$ and the results extrapolated to Fe $\mathrm{X}$, assuming a constant line strength $S$. The first measurements allowed such an extrapolation to Fe X with an uncertainty of about $1 \%$, which after improved experiments on Co could 


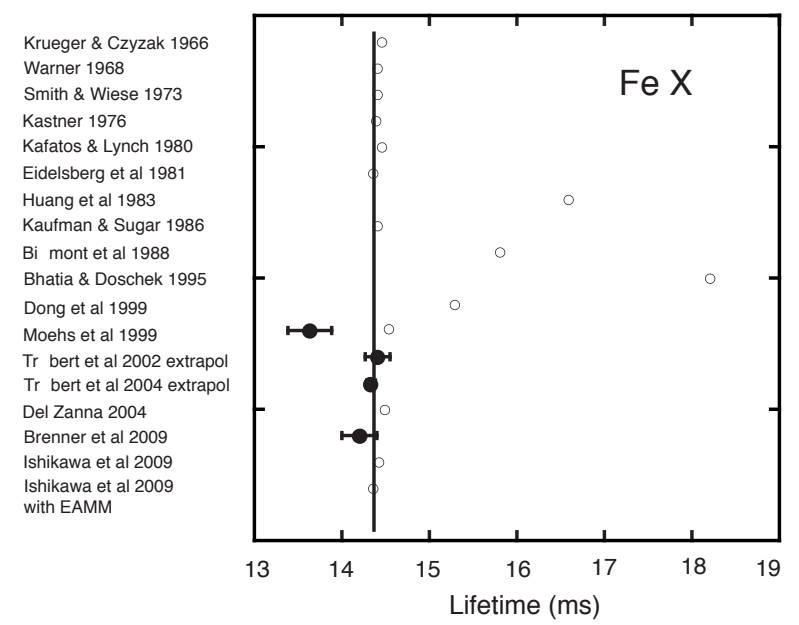

Figure 2. Time line of results for the level lifetime of the ground term fine structure level in Fe X. Open circles: theory, full circles with error bars: experiment. References: Krueger \& Czyzak [69]; Warner [70]; Smith \& Wiese [71]; Kastner [72]; Kafatos \& Lynch [73]; Eidelsberg et al [74]; Huang [75]; Kaufman \& Sugar [76], Biémont et al [77]; Bhatia \& Doschek [78]; Dong et al [79]; Moehs \& Church [58]; Träbert et al [54, 55]; Träbert et al [56]; Del Zanna [47]; Brenner et al [62]; Ishikawa et al (this work). The vertical line marks the expected value based on experimental transition energy and basic model line strength $\mathrm{S}=4 / 3$.

be narrowed down to some $0.4 \%$. Very recently the electron beam ion trap [61] at Heidelberg has yielded a direct measurement result with an uncertainty of slightly more than $1 \%$ [62]. There is excellent agreement of all of the latter experiments with our present calculational result, including the QED correction of the M1 transition operator for the anomalous magnetic moment of the bound electron $[66,67,68]$. It is perhaps amusing to see that our extensive calculation is in good agreement with the result of the practical recipe of determining such M1 transition rates, from a line strength $S$ (in this case close to the nonrelativistic single configuration limit where Racah algebra indicates $S=4 / 3$ ) and the third power of the transition energy. However, our algorithms appear to be the first which can reliably achieve this feat without resorting to semiempirical adjustments. This quality is necessary for testing the validity of atomic structure calculations that are to be applied also to those cases which are more complex.

By far most of the levels depicted in figure 1 have lifetimes of a few nanoseconds or less. Exceptions are the upper one of the fine structure levels of the ground term and three $3 \mathrm{~s}^{2} 3 \mathrm{p}^{4} 3 \mathrm{~d}$ levels that because of their high $J$ values have no E1 decay channels. Theoretical predictions on the lifetimes of such high- $J$ 3d levels have been extremely sparse (although levels with ground state transitions have been covered at several occasions), and several calculations have missed to include the E3 decay branch which in our calculations shows to be significant, especially for the $3 \mathrm{~s}^{2} 3 \mathrm{p}^{4} 3 \mathrm{~d}{ }^{2} \mathrm{G}_{9 / 2}$ level. For measurements with an electrostatic ion trap, the experimenters have made their own calculations [59]. The results are in the same ball park as ours, and they agree more 
or less with their own measurements. However, in detail, our results differ from theirs significantly. Our calculations agree instead with experimental findings from another set of experiments that used a heavy-ion storage ring. That technique has the advantage of a single ion species being stored at a time, which has the potential of cleaner measurements than those using an electrostatic ion trap. The $3 \mathrm{~s}^{2} 3 \mathrm{p}^{4} 3 \mathrm{~d}^{4} \mathrm{~F}_{7 / 2}$ level has been remeasured at another electrostatic ion trap [60], with a result in between the calculations cited in our table 2 .

Overall, we see our lifetime predictions that span a range of eight orders of magnitude well corroborated by experiments. The agreement is best in those cases for which experiment is most highly developed.

\section{Conclusion}

Relativistic MR-MBPT calculations for the strongly correlated system have been carried out to benchmark theoretical accuracy against high-resolution spectroscopic transitions. Term energies of the hitherto unidentified/poorly characterized excited levels of the chlorinelike Fe X are successfully computed with an accuracy on the order of $0.01 \%$. Detailed comparisons of the calculated decay rates and lifetimes of a number of excited levels are made with experiment to critically evaluate recent experiments. The manybody theoretical method has achieved predictive capability in the spectroscopic study of strongly correlated multiple open-shell systems as a valuable theoretical tool for EUV, VUV, and x-ray spectroscopy.

\section{Acknowledgments}

The authors thank Peter Beiersdorfer (Livermore) for advice and support. The work at UPR is supported in part by the Lawrence Livermore National Laboratory under subcontract No. B579693, and the work was also supported by NASA's Astronomy and Physics Research and Analysis Program under contract no. NNH04AA751. ET acknowledges support by the Deutsche Forschungsgemeinschaft (DFG). Some of this work was performed under the auspices of the U.S. Department of Energy by Lawrence Livermore National Laboratory under Contract DE-AC52-07NA27344.

\section{References}

[1] Cheng K-T and Wagner R A 1987 Phys. Rev. A 365435

[2] Blundell S A, Johnson W R and Sapirstein J 1988 Phys. Rev. A 372764

[3] Blundell S A, Sapirstein J and Johnson W R 1992 Phys. Rev. D 451602

[4] Chen M H, Cheng K T and Johnson W R 1993 Phys. Rev. A 473692

[5] Eliav E, Kaldor U and Ishikawa Y 1994 Phys. Rev. A 491724

[6] Avgoustoglou E, Johnson W R, Liu Z W and Sapirstein J 1995 Phys. Rev. A 511196

[7] Eliav E, Kaldor U and Ishikawa Y 1995 Phys. Rev. Lett. 741079

[8] Dzuba V A, Flambaum V V and Kozlov M G 1996 Phys. Rev. A 543948

[9] Beck D R 1997 Phys. Rev. A 562428 
[10] Avgoustoglou E and Beck D R 1993 Phys. Rev. A 574286

[11] Vilkas M J, Ishikawa Y and Koc K 1999 Phys. Rev. A 602808

[12] Safronova M S, Johnson W R and Derevianko A 1999 Phys. Rev. A 604476

[13] Derevianko A, Johnson W R, Safronova M S and Babb J F 1999 Phys. Rev. Lett. 823589

[14] Safronova U I, Johnson W R and Berry H G 2000 Phys. Rev. A 61052503

[15] Ishikawa Y and Vilkas M J 2001 Phys. Rev. A 63042506

[16] Dzuba V A, Flambaum V V and Ginges J S M 2001 Phys. Rev. A 63062101

[17] Savukov I M and Johnson W R 2002 Phys. Rev. A 65042503

[18] Vilkas M J and Ishikawa Y 2003 Phys. Rev. A 68012503

[19] Vilkas M J and Ishikawa Y 2004 Phys. Rev. A 69062503

[20] Dzuba v A 2005 Phys. Rev. A 71032512

[21] Vilkas M J and Ishikawa Y 2005 Phys. Rev. A 72032512

[22] Dzuba V A, Flambaum V V and Safronova M S 2006 Phys. Rev. A 73022112

[23] Safronova U I, Johnson W R and Safronova M S 2007 Phys. Rev. A 76042504

[24] Ishikawa Y and Vilkas M J 2008 Phys. Rev. A 78042501

[25] Blundell S A, Johnson W R, Safronova M S and Safronova U I 2008 Phys. Rev. A 77032507

[26] Grant I P, Mayers D F and Pyper N C 1976 J. Phys. B: At. Mol. Opt. Phys. 92777

[27] Desclaux J P, Cheng K T and Kim Y K 1979 J. Phys. B: At. Mol. Opt. Phys. 123819

[28] Jönsson P, Ynnerman A, Fischer C F, Godefroid M and Olsen J 1996 Phys. Rev. A 534021

[29] Hibbert A and Hansen J E 1994 J. Phys. B: At. Mol. Opt. Phys. 273325

[30] Quiney Q M, Grant I P and Wilson S 1990 J. Phys. B: At. Mol. Opt. Phys. 23 L271

[31] Ishikawa Y and Koc K 1997 Phys. Rev. A 561295

[32] Träbert E, Beiersdorfer P and Chen H 2004 Phys. Rev. A 70032506

[33] Blundell S A 1993 Phys. Rev. A 471790

[34] Vilkas M J, Ishikawa Y and Koc K 1998 Phys. Rev. E 585096

[35] Sucher J 1980 Phys. Rev. A 22348

[36] Mittleman M H 1981 Phys. Rev. A 241167

[37] Indelicato P, Gorceix O and Desclaux J P 1987 J. Phys. B: At. Mol. Opt. Phys. 20651

[38] Kim Y K 1990 in Atomic Processes in Plasmas, AIP Conf. Proc. No. 206 p. 19

[39] Mohr P J 1992 Phys. Rev. A 464421

[40] Malli G L, Da Silva J and Ishikawa Y 1993 Chem. Phys. Lett 20137

[41] Malli G L, Da Silva J and Ishikawa Y 1994 J. Chem. Phys. 1016829

[42] Ishikawa Y, Quiney H M and Malli G L 1991 Phys. Rev. A 433270

[43] Ishikawa Y, Koc K and Schwarz W H E 1997 Chem. Phys. 225239

[44] Grant I P 1974 J. Phys. B: At. Mol. Opt. Phys. 71458

[45] Johnson W R, Plante D R and Sapirstein J 1995 Adv. At. Mol. Opt. Phys. 35255

[46] Fuhr J R et al., NIST Atomic Spectra Database Ver. 3.1.00, NIST Physical Reference Data. Available on line at http://physics.nist.gov/PhysRefDat/ASD/index.html.

[47] Del Zanna G, Berrington K A and Mason H E 2004 Astron. Astrophys. 422731

[48] Aggarwal K M and Keenan F P 2005 Astron. Astrophys. 4391215

[49] Keenan F P, Jess D B, Aggarwal K M, Thomas R J, Brosius J W and Davila J M 2008 Mon. Not. R. Astron. Soc. 389939

[50] Träbert E 1996 J. Phys. B: At. Mol. Opt. Phys. 29 L217

[51] Biémont É and Träbert E 2000 J. Phys. B: At. Mol. Opt. Phys. 332939

[52] Lauer S, Liebel H, Vollweiler F, Schmoranzer H, Lagutin B M, Demekhin Ph V, Petrov I D and Sukhorukov V L 1999 J. Phys. B: At. Mol. Opt. Phys. 32, 2015

[53] Träbert E, Gwinner G, Wolf A, Knystautas E J, Garnir H-P and Tordoir X 2002 J. Phys. B: At. Mol. Opt. Phys. 35671

[54] Träbert E, Saathoff G and Wolf A 2004 J. Phys. B: At. Mol. Opt. Phys. 37945

[55] Träbert E 2004 Astron. Astrophys. 415 L39

[56] Träbert E, Reinhardt S, Hoffmann J and Wolf A 2006 J. Phys. B: At. Mol. Opt. Phys. 39945 
[57] Moehs D P, Bhatti M I and Church D A 2001 Phys. Rev. A 63032515

[58] Moehs D P and Church D A 1999 Astrophys. J 516 L111

[59] Moehs D P, Church D A, Bhatti M I and Perger W F 2000 Phys. Rev. Lett. 8538

[60] Smith S J, Chutjian A and Lozano J A 2005 Phys. Rev. A 72062504

[61] Träbert E 2008 Can. J. Phys. 8673

[62] Brenner G, Crespo L'opez-Urrutia J R, Bernitt S, Fischer D, Ginzel R, Kubiček K, Mäckel V, Mokler P H, Siman M C and Ullrich J 2009 Astrophys. J. (submitted)

[63] Kohstall C, Fritzsche S, Fricke B, Sepp W-D and Träbert E 1999 Phys. Scr. T 80482

[64] Pelan J C and Berrington K A 2001 Astron. Astrophys. 365258

[65] Aggarwal K M and Keenan F P 2004 Astron. Astrophys. 427763

[66] Lapierre A, Jentschura U D, Crespo López-Urrutia J R, Braun J, Brenner G, Bruhns H, Fischer D, González Martínez A J, Harman Z, Johnson W R, Keitel C H, Mironov V, Osborne C J, Sikler G, Soria Orts R, Shabaev V, Tawara H, Tupitsyn I I, Ullrich J and Volotka A 2005 Phys. Rev. Lett. 95183001

[67] Lapierre A, Crespo López-Urrutia J R, Braun J, Brenner G, Bruhns H, Fischer D, GonzálezMartínez A J, Mironov V, Osborne C J, Sikler G, Soria Orts R, Tawara H, Ullrich J, Shabaev V M, Tupitsyn I I and Volotka A 2006 Phys. Rev. A 73052507

[68] Nemouchi M and Godefroid M 2009 J. Phys. B: At. Mol. Opt. Phys. 42175002

[69] Krueger T K and Czyzak S J 1966 Astroph. J. 144 1194; erratum 1967 Astroph. J. 149237

[70] Warner B 1968 Z. Astrophys. 69399

[71] Smith M W and Wiese W L 1973 J. Phys. Chem. Ref. Data 285

[72] Kastner S O 1976 Sol. Phys. 46179

[73] Kafatos M and Lynch J P 1980, Astroph. J. Suppl. 42611

[74] Eidelsberg M, Crifo-Magnant F and Zeippen C J 1981 Astron. Astroph. Suppl. 43455

[75] Huang K-N, Kim Y-K, Cheng K T and Desclaux J P 1983 At. Data Nucl. Data Tables 28355

[76] Kaufman V and Sugar J 1986 J. Phys. Chem. Ref. Data 15321

[77] Biémont E, Cowan R D and Hansen J E 1988 Phys. Scr. 37850

[78] Bhatia A K and Doschek G A 1995 At. Data Nucl. Data Tables 6097

[79] Dong C Z, Fritzsche S, Fricke B and Sepp W-D 1999 Mon. Not. R. Astron. Soc. 397809

[80] Träbert E, Calamai A G, Gwinner G, Knystautas E J, Pinnington E H and Wolf A 2003 J. Phys. B: At. Mol. Opt. Phys. 361129 
Table 1: Comparison of MR-MBPT calculated term energies (in $\mathrm{cm}^{-1}$ ) (this work) with experiment [46, 47] and with other theory [65]. The numbers in parentheses indicate the deviation (in percent) from our calculated results.

\begin{tabular}{|c|c|c|c|c|c|}
\hline Configuration & Term & MR-MP2 & NIST & Del Zanna et al. & Aggarwal et al. \\
\hline $13 s^{2} 3 p^{5}$ & & 0 & 0 & 0 & 0 \\
\hline $23 \mathrm{~s}^{2} 3 \mathrm{p}^{5}$ & ${ }^{2} \mathrm{P}_{1 / 2}^{0}$ & 15683 & $15683(0.00)$ & $15683(0.00)$ & $15649(-0.22)$ \\
\hline $33 \mathrm{~s} 3 \mathrm{p}^{6}$ & ${ }^{2} \mathrm{~S}_{1 / 2}$ & 288932 & $289249(+0.11)$ & $289236(+0.11)$ & $285580(-1.16)$ \\
\hline $43 \mathrm{~s}^{2} 3 \mathrm{p}^{4}\left({ }^{3} \mathrm{P}\right) 3 \mathrm{~d}$ & ${ }^{4} \mathrm{D}_{7 / 2}$ & 388814 & $388709(-0.03)$ & $388708(+0.03)$ & $389216(+0.10)$ \\
\hline $53 \mathrm{~s}^{2} 3 \mathrm{p}^{4}\left({ }^{3} \mathrm{P}\right) 3 \mathrm{~d}$ & ${ }^{4} \mathrm{D}_{5 / 2}$ & 388832 & $388709(-0.03)$ & $388714(-0.03)$ & $389271(+0.11)$ \\
\hline $63 \mathrm{~s}^{2} 3 \mathrm{p}^{4}\left({ }^{3} \mathrm{P}\right) 3 \mathrm{~d}$ & ${ }^{4} \mathrm{D}_{3 / 2}$ & 390080 & $390050(-0.01)$ & $390019(-0.02)$ & $390522(+0.11)$ \\
\hline $73 \mathrm{~s}^{2} 3 \mathrm{p}^{4}\left({ }^{3} \mathrm{P}\right) 3 \mathrm{~d}$ & ${ }^{4} \mathrm{D}_{1 / 2}$ & 391617 & $391555(-0.02)$ & $391557(-0.02)$ & $392023(+0.10)$ \\
\hline $83 \mathrm{~s}^{2} 3 \mathrm{p}^{4}\left({ }^{3} \mathrm{P}\right) 3 \mathrm{~d}$ & ${ }^{4} \mathrm{~F}_{9 / 2}$ & 417763 & $417653(-0.03)$ & $417652(-0.03)$ & $421468(+0.89)$ \\
\hline $93 \mathrm{~s}^{2} 3 \mathrm{p}^{4}\left({ }^{1} \mathrm{D}\right) 3 \mathrm{~d}$ & ${ }^{2} \mathrm{P}_{1 / 2}$ & 419904 & & $414249(-1.35)$ & $423674(+0.90)$ \\
\hline $103 \mathrm{~s}^{2} 3 \mathrm{p}^{4}\left({ }^{3} \mathrm{P}\right) 3 \mathrm{~d}$ & ${ }^{4} \mathrm{~F}_{\tau / 2}$ & 422918 & $422795(-0.03)$ & $422785(-0.03)$ & $426615(+0.87)$ \\
\hline $113 \mathrm{~s}^{2} 3 \mathrm{p}^{4}\left({ }^{3} \mathrm{P}\right) 3 \mathrm{~d}$ & ${ }^{4} \mathrm{~F}_{5 / 2}$ & 426814 & $426763(-0.01)$ & $426260(-0.13)$ & $430390(+0.84)$ \\
\hline $123 \mathrm{~s}^{2} 3 \mathrm{p}^{4}\left({ }^{3} \mathrm{P}\right) 3 \mathrm{~d}$ & ${ }^{4} \mathrm{~F}_{3 / 2}$ & 428088 & $428298(+0.05)$ & $427604(-0.11)$ & $431761(+0.86)$ \\
\hline $133 \mathrm{~s}^{2} 3 \mathrm{p}^{4}\left({ }^{1} \mathrm{D}\right) 3 \mathrm{~d}$ & ${ }^{2} \mathrm{P}_{3 / 2}$ & 428432 & $431928(+0.81)$ & $422844(-1.30)$ & $432091(+0.85)$ \\
\hline $143 \mathrm{~s}^{2} 3 \mathrm{p}^{4}\left({ }^{1} \mathrm{D}\right) 3 \mathrm{~d}$ & ${ }^{2} \mathrm{D}_{3 / 2}$ & 434821 & $434614(-0.05)$ & $433088(-0.40)$ & $438313(+0.80)$ \\
\hline $153 \mathrm{~s}^{2} 3 \mathrm{p}^{4}\left({ }^{3} \mathrm{P}\right) 3 \mathrm{~d}$ & ${ }^{4} \mathrm{P}_{1 / 2}$ & 435062 & $434800(-0.06)$ & $433526(-0.35)$ & $438861(+0.87)$ \\
\hline $163 \mathrm{~s}^{2} 3 \mathrm{p}^{4}\left({ }^{3} \mathrm{P}\right) 3 \mathrm{~d}$ & ${ }^{4} \mathrm{P}_{3 / 2}$ & 439981 & & $438168(-0.41)$ & $443723(+0.85)$ \\
\hline $173 \mathrm{~s}^{2} 3 \mathrm{p}^{4}\left({ }^{3} \mathrm{P}\right) 3 \mathrm{~d}$ & ${ }^{2} \mathrm{~F}_{7 / 2}$ & 440881 & $440840(-0.01)$ & $440839(-0.01)$ & $446806(+1.34)$ \\
\hline $183 \mathrm{~s}^{2} 3 \mathrm{p}^{4}\left({ }^{3} \mathrm{P}\right) 3 \mathrm{~d}$ & ${ }^{4} \mathrm{P}_{5 / 2}$ & 442110 & $441853(-0.06)$ & $440125(-0.45)$ & $445698(+0.81)$ \\
\hline $193 \mathrm{~s}^{2} 3 \mathrm{p}^{4}\left({ }^{1} \mathrm{D}\right) 3 \mathrm{~d}$ & ${ }^{2} \mathrm{D}_{5 / 2}$ & 444377 & & $442760(-0.36)$ & $448123(+0.84)$ \\
\hline $203 \mathrm{~s}^{2} 3 \mathrm{p}^{4}\left({ }^{1} \mathrm{D}\right) 3 \mathrm{~d}$ & ${ }^{2} G_{9 / 2}$ & 450859 & $450751(-0.02)$ & $450754(-0.02)$ & $456639(+1.28)$ \\
\hline $213 \mathrm{~s}^{2} 3 \mathrm{p}^{4}\left({ }^{1} \mathrm{D}\right) 3 \mathrm{~d}$ & ${ }^{2} G_{7 / 2}$ & 451182 & $451084(-0.02)$ & $451083(-0.02)$ & $457166(+1.33)$ \\
\hline $223 \mathrm{~s}^{2} 3 \mathrm{p}^{4}\left({ }^{3} \mathrm{P}\right) 3 \mathrm{~d}$ & ${ }^{2} \mathrm{~F}_{5 / 2}$ & 454059 & $452730(-0.29)$ & $454036(-0.01)$ & $460183(+1.35)$ \\
\hline $233 \mathrm{~s}^{2} 3 \mathrm{p}^{4}\left({ }^{1} \mathrm{D}\right) 3 \mathrm{~d}$ & ${ }^{2} \mathrm{~F}_{5 / 2}$ & 482238 & $476699(-1.15)$ & $482046(-0.04)$ & $490131(+1.64)$ \\
\hline $243 \mathrm{~s}^{2} 3 \mathrm{p}^{4}\left({ }^{1} \mathrm{D}\right) 3 \mathrm{~d}$ & ${ }^{2} \mathrm{~F}_{7 / 2}$ & 486162 & $485983(-0.04)$ & $485982(-0.04)$ & $494037(+1.62)$ \\
\hline $253 \mathrm{~s}^{2} 3 \mathrm{p}^{4}\left({ }^{1} \mathrm{~S}\right) 3 \mathrm{~d}$ & ${ }^{2} \mathrm{D}_{3 / 2}$ & 511975 & $511800(-0.03)$ & $511992(+0.00)$ & $518432(+1.26)$ \\
\hline $263 \mathrm{~s}^{2} 3 \mathrm{p}^{4}\left({ }^{1} \mathrm{~S}\right) 3 \mathrm{~d}$ & ${ }^{2} \mathrm{D}_{5 / 2}$ & 516374 & & $516222(-0.03)$ & $522547(+1.20)$ \\
\hline $273 \mathrm{~s}^{2} 3 \mathrm{p}^{4}\left({ }^{1} \mathrm{D}\right) 3 \mathrm{~d}$ & ${ }^{2} \mathrm{~S}_{1 / 2}$ & 542316 & $541879(-0.08)$ & $541897(-0.08)$ & $551189(+1.64)$ \\
\hline $283 \mathrm{~s}^{2} 3 \mathrm{p}^{4}\left({ }^{3} \mathrm{P}\right) 3 \mathrm{~d}$ & ${ }^{2} \mathrm{P}_{3 / 2}$ & 564980 & $564198(-0.14)$ & $564208(-0.14)$ & $578612(+2.41)$ \\
\hline $293 \mathrm{~s}^{2} 3 \mathrm{p}^{4}\left({ }^{3} \mathrm{P}\right) 3 \mathrm{~d}$ & ${ }^{2} \mathrm{P}_{1 / 2}$ & 570654 & $569985(-0.12)$ & $569882(-0.14)$ & $584274(+2.39)$ \\
\hline $303 \mathrm{~s}^{2} 3 \mathrm{p}^{4}\left({ }^{3} \mathrm{P}\right) 3 \mathrm{~d}$ & ${ }^{2} \mathrm{D}_{5 / 2}$ & 573699 & $572954(-0.13)$ & $572964(-0.13)$ & $586304(+2.20)$ \\
\hline $313 \mathrm{~s}^{2} 3 \mathrm{p}^{4}\left({ }^{3} \mathrm{P}\right) 3 \mathrm{~d}$ & ${ }^{2} \mathrm{D}_{3 / 2}$ & 586963 & $586244(-0.12)$ & $586254(-0.12)$ & $599528(+2.14)$ \\
\hline $323 \mathrm{~s} 3 \mathrm{p}^{5}\left({ }^{3} \mathrm{P}\right) 3 \mathrm{~d}$ & ${ }^{4} \mathrm{P}_{1 / 2}^{\circ}$ & 666185 & & $661175(-0.75)$ & $683137(+2.54)$ \\
\hline $333 \mathrm{~s} 3 \mathrm{p}^{5}\left({ }^{3} \mathrm{P}\right) 3 \mathrm{~d}$ & ${ }^{4} \mathrm{P}_{3 / 2}^{\circ}$ & 668722 & & $663782(-0.74)$ & $685694(+2.54)$ \\
\hline
\end{tabular}


Table 1: (continued)

\begin{tabular}{|c|c|c|c|c|c|}
\hline Configuration & Term & MR-MP2 & NIST & Del Zanna et al. & Aggarwal et al. \\
\hline $343 \mathrm{~s} 3 \mathrm{p}^{5}\left({ }^{3} \mathrm{P}\right) 3 \mathrm{~d}$ & ${ }^{4} \mathrm{P}_{5 / 2}^{o}$ & 673362 & & $668467(-0.73)$ & $690302(+2.52)$ \\
\hline $353 \mathrm{~s} 3 \mathrm{p}^{5}\left({ }^{3} \mathrm{P}\right) 3 \mathrm{~d}$ & ${ }^{4} \mathrm{~F}_{9 / 2}^{0}$ & 696427 & $696661(+0.03)$ & $694225(-0.32)$ & $718955(+3.23)$ \\
\hline $363 \mathrm{~s} 3 \mathrm{p}^{5}\left({ }^{3} \mathrm{P}\right) 3 \mathrm{~d}$ & ${ }^{4} \mathrm{~F}_{7 / 2}^{o}$ & 699271 & $699492(+0.03)$ & $697016(-0.32)$ & $721753(+3.22)$ \\
\hline $373 \mathrm{~s} 3 \mathrm{p}^{5}\left({ }^{3} \mathrm{P}\right) 3 \mathrm{~d}$ & ${ }^{4} \mathrm{~F}_{5 / 2}^{0}$ & 702393 & $702585(+0.03)$ & $700011(-0.34)$ & $724749(+3.18)$ \\
\hline $383 \mathrm{~s} 3 \mathrm{p}^{5}\left({ }^{3} \mathrm{P}\right) 3 \mathrm{~d}$ & ${ }^{4} \mathrm{~F}_{3 / 2}^{0}$ & 705124 & $705430(+0.04)$ & $702749(-0.34)$ & $727481(+3.17)$ \\
\hline $393 \mathrm{~s} 3 \mathrm{p}^{5}\left({ }^{3} \mathrm{P}\right) 3 \mathrm{~d}$ & ${ }^{4} \mathrm{D}_{7 / 2}^{\circ}$ & 727570 & & $726123(-0.20)$ & $751744(+3.32)$ \\
\hline $403 \mathrm{~s} 3 \mathrm{p}^{5}\left({ }^{3} \mathrm{P}\right) 3 \mathrm{~d}$ & ${ }^{4} \mathrm{D}_{1 / 2}^{\circ}$ & 728941 & & $727262(-0.23)$ & $753127(+3.32)$ \\
\hline $413 \mathrm{~s} 3 \mathrm{p}^{5}\left({ }^{3} \mathrm{P}\right) 3 \mathrm{~d}$ & ${ }^{4} \mathrm{D}_{5 / 2}^{0}$ & 729333 & & $727681(-0.23)$ & $753278(+3.31)$ \\
\hline $423 \mathrm{~s} 3 \mathrm{p}^{5}\left({ }^{3} \mathrm{P}\right) 3 \mathrm{~d}$ & ${ }^{4} \mathrm{D}_{3 / 2}^{\circ}$ & 729529 & & $727821(-0.23)$ & $753654(+3.31)$ \\
\hline $433 \mathrm{~s} 3 \mathrm{p}^{5}\left({ }^{3} \mathrm{P}\right) 3 \mathrm{~d}$ & ${ }^{2} \mathrm{~F}_{7 / 2}^{0}$ & 737196 & & $737368(+0.02)$ & $760128(+3.11)$ \\
\hline $443 \mathrm{~s} 3 \mathrm{p}^{5}\left({ }^{3} \mathrm{P}\right) 3 \mathrm{~d}$ & ${ }^{2} \mathrm{D}_{5 / 2}^{\circ}$ & 741411 & & $741260(-0.02)$ & $775448(+4.59)$ \\
\hline $453 \mathrm{~s} 3 \mathrm{p}^{5}\left({ }^{3} \mathrm{P}\right) 3 \mathrm{~d}$ & ${ }^{2} \mathrm{D}_{3 / 2}^{\circ}$ & 745921 & & $745812(-0.01)$ & $773758(+3.73)$ \\
\hline $463 \mathrm{~s} 3 \mathrm{p}^{5}\left({ }^{3} \mathrm{P}\right) 3 \mathrm{~d}$ & ${ }^{2} \mathrm{~F}_{5 / 2}^{0}$ & 749519 & & $749382(-0.02)$ & $766614(+2.28)$ \\
\hline $473 \mathrm{~s} 3 \mathrm{p}^{5}\left({ }^{1} \mathrm{P}\right) 3 \mathrm{~d}$ & ${ }^{2} \mathrm{P}_{1 / 2}^{\circ}$ & 759703 & & $760037(-0.04)$ & $784164(+3.22)$ \\
\hline $483 \mathrm{~s} 3 \mathrm{p}^{5}\left({ }^{1} \mathrm{P}\right) 3 \mathrm{~d}$ & ${ }^{2} \mathrm{P}_{3 / 2}^{\circ}$ & 767418 & & $767867(+0.06)$ & $792369(+3.25)$ \\
\hline $493 \mathrm{~s}^{2} 3 \mathrm{p}^{3}\left({ }^{4} \mathrm{~S}\right) 3 \mathrm{~d}^{2}\left({ }^{3} \mathrm{~F}\right)$ & ${ }^{6} \mathrm{~F}_{1 / 2}^{0}$ & 782254 & & & $798032(+2.02)$ \\
\hline $503 \mathrm{~s}^{2} 3 \mathrm{p}^{3}\left({ }^{4} \mathrm{~S}\right) 3 \mathrm{~d}^{2}\left({ }^{3} \mathrm{~F}\right)$ & ${ }^{6} \mathrm{~F}_{3 / 2}^{0}$ & 782413 & & & $798185(+2.02)$ \\
\hline $513 \mathrm{~s}^{2} 3 \mathrm{p}^{3}\left({ }^{4} \mathrm{~S}\right) 3 \mathrm{~d}^{2}\left({ }^{3} \mathrm{~F}\right)$ & ${ }^{6} \mathrm{~F}_{5 / 2}^{0}$ & 782765 & & & $798471(+2.01)$ \\
\hline $523 \mathrm{~s}^{2} 3 \mathrm{p}^{3}\left({ }^{4} \mathrm{~S}\right) 3 \mathrm{~d}^{2}\left({ }^{3} \mathrm{~F}\right)$ & ${ }^{6} \mathrm{~F}_{7 / 2}^{0}$ & 783210 & & & $798920(+2.01)$ \\
\hline $533 \mathrm{~s}^{2} 3 \mathrm{p}^{3}\left({ }^{4} \mathrm{~S}\right) 3 \mathrm{~d}^{2}\left({ }^{3} \mathrm{~F}\right)$ & ${ }^{6} \mathrm{~F}_{9 / 2}^{0}$ & 783811 & & & $799568(+2.01)$ \\
\hline $543 \mathrm{~s}^{2} 3 \mathrm{p}^{3}\left({ }^{4} \mathrm{~S}\right) 3 \mathrm{~d}^{2}\left({ }^{3} \mathrm{~F}\right)$ & ${ }^{6} \mathrm{~F}_{11 / 2}^{0}$ & 784592 & & & $800369(+2.01)$ \\
\hline $553 \mathrm{~s} 3 \mathrm{p}^{5}\left({ }^{1} \mathrm{P}\right) 3 \mathrm{~d}$ & ${ }^{2} \mathrm{~F}_{5 / 2}^{0}$ & 786586 & & $789807(+0.41)$ & $814657(+3.57)$ \\
\hline $563 \mathrm{~s} 3 \mathrm{p}^{5}\left({ }^{1} \mathrm{P}\right) 3 \mathrm{~d}$ & ${ }^{2} \mathrm{~F}_{7 / 2}^{0}$ & 790379 & & $793645(+0.41)$ & $818926(+3.61)$ \\
\hline $573 \mathrm{~s}^{2} 3 \mathrm{p}^{3}\left({ }^{4} \mathrm{~S}\right) 3 \mathrm{~d}^{2}\left({ }^{3} \mathrm{P}\right)$ & ${ }^{6} \mathrm{P}_{3 / 2}^{0}$ & 806147 & & & $825916(+2.45)$ \\
\hline $583 \mathrm{~s}^{2} 3 \mathrm{p}^{3}\left({ }^{4} \mathrm{~S}\right) 3 \mathrm{~d}^{2}\left({ }^{3} \mathrm{P}\right)$ & ${ }^{6} \mathrm{P}_{5 / 2}^{0}$ & 806509 & & & $826267(+2.45)$ \\
\hline $593 \mathrm{~s}^{2} 3 \mathrm{p}^{3}\left({ }^{4} \mathrm{~S}\right) 3 \mathrm{~d}^{2}\left({ }^{3} \mathrm{P}\right)$ & ${ }^{6} \mathrm{P}_{7 / 2}^{\circ}$ & 806796 & & & $826574(+2.45)$ \\
\hline $603 \mathrm{~s} 3 \mathrm{p}^{5}\left({ }^{1} \mathrm{P}\right) 3 \mathrm{~d}$ & ${ }^{2} \mathrm{D}_{3 / 2}^{\circ}$ & 813997 & & $818347(+0.53)$ & $842530(+3.51)$ \\
\hline $613 \mathrm{~s} 3 \mathrm{p}^{5}\left({ }^{1} \mathrm{P}\right) 3 \mathrm{~d}$ & ${ }^{2} \mathrm{D}_{5 / 2}^{\circ}$ & 815436 & & $819974(+0.56)$ & $844220(+3.53)$ \\
\hline $623 \mathrm{~s}^{2} 3 \mathrm{p}^{3}\left({ }^{4} \mathrm{~S}\right) 3 \mathrm{~d}^{2}\left({ }^{1} \mathrm{D}\right)$ & ${ }^{4} \mathrm{D}_{7 / 2}^{\circ}$ & 826951 & & & $851605(+2.98)$ \\
\hline $633 \mathrm{~s}^{2} 3 \mathrm{p}^{3}\left({ }^{4} \mathrm{~S}\right) 3 \mathrm{~d}^{2}\left({ }^{1} \mathrm{D}\right)$ & ${ }^{4} \mathrm{D}_{1 / 2}^{\circ}$ & 827233 & & & $854305(+3.27)$ \\
\hline $643 \mathrm{~s}^{2} 3 \mathrm{p}^{3}\left({ }^{4} \mathrm{~S}\right) 3 \mathrm{~d}^{2}\left({ }^{1} \mathrm{D}\right)$ & ${ }^{4} \mathrm{D}_{5 / 2}^{\circ}$ & 827623 & & & $852439(+3.00)$ \\
\hline $653 \mathrm{~s}^{2} 3 \mathrm{p}^{3}\left({ }^{4} \mathrm{~S}\right) 3 \mathrm{~d}^{2}\left({ }^{1} \mathrm{D}\right)$ & ${ }^{4} \mathrm{D}_{3 / 2}^{\circ}$ & 828262 & & & $853032(+2.99)$ \\
\hline $663 \mathrm{~s}^{2} 3 \mathrm{p}^{3}\left({ }^{2} \mathrm{D}\right) 3 \mathrm{~d}^{2}\left({ }^{3} \mathrm{P}\right)$ & ${ }^{4} \mathrm{P}_{1 / 2}^{\circ}$ & 829640 & & & $855413(+3.11)$ \\
\hline $673 \mathrm{~s}^{2} 3 \mathrm{p}^{3}\left({ }^{2} \mathrm{D}\right) 3 \mathrm{~d}^{2}\left({ }^{3} \mathrm{P}\right)$ & ${ }^{4} \mathrm{~F}_{3 / 2}^{0}$ & 830297 & & & $857345(+3.26)$ \\
\hline $683 \mathrm{~s}^{2} 3 \mathrm{p}^{3}\left({ }^{2} \mathrm{D}\right) 3 \mathrm{~d}^{2}\left({ }^{3} \mathrm{~F}\right)$ & ${ }^{4} \mathrm{G}_{5 / 2}^{0}$ & 832558 & & & $855358(+2.74)$ \\
\hline $693 \mathrm{~s}^{2} 3 \mathrm{p}^{3}\left({ }^{2} \mathrm{D}\right) 3 \mathrm{~d}^{2}\left({ }^{3} \mathrm{~F}\right)$ & ${ }^{4} \mathrm{G}_{7 / 2}^{0}$ & 832568 & & & $856456(+2.87)$ \\
\hline
\end{tabular}


Table 1: (continued)

\begin{tabular}{|c|c|c|c|c|c|}
\hline Configuration & Term & MR-MP2 & NIST & Del Zanna et al. & Aggarwal et al \\
\hline $703 \mathrm{~s}^{2} 3 \mathrm{p}^{3}\left({ }^{2} \mathrm{D}\right) 3 \mathrm{~d}^{2}\left({ }^{3} \mathrm{P}\right)$ & ${ }^{4} \mathrm{P}_{3 / 2}^{\circ}$ & 832846 & & & $858936(+3.13)$ \\
\hline $713 \mathrm{~s}^{2} 3 \mathrm{p}^{3}\left({ }^{2} \mathrm{D}\right) 3 \mathrm{~d}^{2}\left({ }^{3} \mathrm{P}\right)$ & ${ }^{4} \mathrm{~F}_{5 / 2}^{0}$ & 833048 & & & $860911(+3.34)$ \\
\hline $723 \mathrm{~s}^{2} 3 \mathrm{p}^{3}\left({ }^{2} \mathrm{D}\right) 3 \mathrm{~d}^{2}\left({ }^{3} \mathrm{~F}\right)$ & ${ }^{4} \mathrm{G}_{9 / 2}^{0}$ & 834705 & & & $858475(+2.85)$ \\
\hline $733 \mathrm{~s}^{2} 3 \mathrm{p}^{3}\left({ }^{2} \mathrm{D}\right) 3 \mathrm{~d}^{2}\left({ }^{3} \mathrm{~F}\right)$ & ${ }^{4} \mathrm{G}_{11 / 2}^{0}$ & 836759 & & & $860626(+2.85)$ \\
\hline $743 \mathrm{~s}^{2} 3 \mathrm{p}^{3}\left({ }^{2} \mathrm{D}\right) 3 \mathrm{~d}^{2}\left({ }^{3} \mathrm{P}\right)$ & ${ }^{4} \mathrm{P}_{5 / 2}^{o}$ & 836815 & & & $862436(+3.06)$ \\
\hline $753 \mathrm{~s}^{2} 3 \mathrm{p}^{3}\left({ }^{2} \mathrm{D}\right) 3 \mathrm{~d}^{2}\left({ }^{3} \mathrm{P}\right)$ & ${ }^{4} \mathrm{~F}_{7 / 2}^{0}$ & 839827 & & & $864873(+2.98)$ \\
\hline $763 \mathrm{~s}^{2} 3 \mathrm{p}^{3}\left({ }^{2} \mathrm{D}\right) 3 \mathrm{~d}^{2}\left({ }^{3} \mathrm{P}\right)$ & ${ }^{4} \mathrm{~F}_{9 / 2}^{0}$ & 842791 & & & $868044(+3.00)$ \\
\hline $773 \mathrm{~s}^{2} 3 \mathrm{p}^{3}\left({ }^{2} \mathrm{D}\right) 3 \mathrm{~d}^{2}\left({ }^{3} \mathrm{~F}\right)$ & ${ }^{4} \mathrm{H}_{7 / 2}^{0}$ & 851673 & & & $875111(+2.75)$ \\
\hline $783 \mathrm{~s}^{2} 3 \mathrm{p}^{3}\left({ }^{2} \mathrm{D}\right) 3 \mathrm{~d}^{2}\left({ }^{3} \mathrm{~F}\right)$ & ${ }^{4} \mathrm{H}_{9 / 2}^{0}$ & 852949 & & & $876066(+2.71)$ \\
\hline $793 \mathrm{~s}^{2} 3 \mathrm{p}^{3}\left({ }^{2} \mathrm{D}\right) 3 \mathrm{~d}^{2}\left({ }^{3} \mathrm{~F}\right)$ & ${ }^{4} \mathrm{H}_{11 / 2}^{\circ}$ & 854465 & & & $877503(+2.70)$ \\
\hline $803 \mathrm{~s}^{2} 3 \mathrm{p}^{3}\left({ }^{2} \mathrm{D}\right) 3 \mathrm{~d}^{2}\left({ }^{1} \mathrm{D}\right)$ & ${ }^{2} \mathrm{~F}_{7 / 2}^{o}$ & 855953 & & & $883967(+3.27)$ \\
\hline $813 \mathrm{~s} 3 \mathrm{p}^{5}\left({ }^{3} \mathrm{P}\right) 3 \mathrm{~d}$ & ${ }^{2} \mathrm{P}_{1 / 2}^{o}$ & 858374 & & $864859(+0.75)$ & $900482(+4.91)$ \\
\hline $823 \mathrm{~s}^{2} 3 \mathrm{p}^{3}\left({ }^{2} \mathrm{D}\right) 3 \mathrm{~d}^{2}\left({ }^{1} \mathrm{D}\right)$ & ${ }^{2} \mathrm{D}_{3 / 2}^{\circ}$ & 858582 & & & $891188(+3.80)$ \\
\hline $833 \mathrm{~s} 3 \mathrm{p}^{5}\left({ }^{3} \mathrm{P}\right) 3 \mathrm{~d}$ & ${ }^{2} \mathrm{P}_{3 / 2}^{\circ}$ & 859830 & & $865405(+0.65)$ & $901152(+4.81)$ \\
\hline $843 \mathrm{~s}^{2} 3 \mathrm{p}^{3}\left({ }^{2} \mathrm{D}\right) 3 \mathrm{~d}^{2}\left({ }^{3} \mathrm{~F}\right)$ & ${ }^{4} \mathrm{G}_{5 / 2}^{0}$ & 860015 & & & $898342(+4.46)$ \\
\hline
\end{tabular}


Table 2: E1/M1/E2/M2/E3 transition probabilities $\left(\mathrm{s}^{-1}\right)$ and lifetimes $\tau(\mathrm{ms})$ of the states arising from the $3 \mathrm{~s}^{2} 3 \mathrm{p}^{5}, 3 \mathrm{~s}^{2} 3 \mathrm{p}^{4} 3 \mathrm{~d}$, and $3 \mathrm{~s} 3 \mathrm{p}^{6}$ configurations. $a(b)$ stands for $a \times 10^{b}$.

\begin{tabular}{|c|c|c|c|c|c|}
\hline \multirow[t]{2}{*}{$\mathcal{J}_{\text {upper }} \rightarrow \mathcal{J}_{\text {lower }}$} & \multirow[t]{2}{*}{$\lambda(n m)$} & \multirow[t]{2}{*}{$A_{j i}\left(s^{-1}\right)$} & \multirow[t]{2}{*}{ Exp. $\tau(m s)$} & \multicolumn{2}{|c|}{ Theoretical $\tau(m s)$} \\
\hline & & & & Previous & MR-MBPT \\
\hline \multirow{4}{*}{${ }^{2} \mathrm{P}_{1 / 2}^{o} \rightarrow{ }^{2} \mathrm{P}_{3 / 2}^{o}$} & 637.633 & M1: 69.28 & $14.41 \pm 0.14^{a}$ & $14.54^{b, c}$ & 14.43 \\
\hline & & $\mathrm{E} 2: 7.27(-3)$ & $13.64 \pm 0.25^{b, c}$ & $14.51^{d}$ & $14.37^{e}$ \\
\hline & & & $14.33 \pm 0.05^{f}$ & & \\
\hline & & & $14.2 \pm 0.2^{g}$ & & \\
\hline \multirow{3}{*}{$\begin{aligned}{ }^{2} \mathrm{~S}_{1 / 2} & \rightarrow{ }^{2} \mathrm{P}_{1 / 2}^{\mathrm{o}} \\
& \rightarrow{ }^{2} \mathrm{P}_{3 / 2}^{\mathrm{o}}\end{aligned}$} & 36.597 & $\mathrm{E} 1: 1.26(+9)$ & $2.7 \pm 0.2(-7)^{h}$ & $2.3(-7)^{i}$ & $2.41(-7)$ \\
\hline & 34.610 & E1: $2.89(+9)$ & & $2.42(-7)^{j}$ & \\
\hline & & & & $2.34(-7)^{d}$ & \\
\hline \multirow{3}{*}{$\begin{aligned}{ }^{4} \mathrm{~F}_{9 / 2} & \rightarrow{ }^{4} \mathrm{D}_{5 / 2} \\
& \rightarrow{ }^{4} \mathrm{D}_{\overline{7} / 2}\end{aligned}$} & 345.650 & $\mathrm{E} 2: 6.90(-3)$ & $85.7 \pm 9.2^{k}$ & $80.2^{k}$ & 108.0 \\
\hline & 345.435 & $\mathrm{E} 2: 3.50(-3)$ & $110 \pm 5^{l}$ & $77.3^{d}$ & \\
\hline & & M1: 9.23 & & & \\
\hline$\rightarrow{ }^{2} \mathrm{P}_{3 / 2}^{\mathrm{o}}$ & 23.937 & E3: $2.22(-2)$ & & & \\
\hline \multirow[t]{2}{*}{${ }^{4} \mathrm{~F}_{7 / 2} \rightarrow{ }^{4} \mathrm{~F}_{9 / 2}$} & 1939.864 & M1: 4.31 & $93 \pm 30^{k}$ & $80^{k}$ & 66.49 \\
\hline & & $\mathrm{E} 2: 1.95(-5)$ & $73.0 \pm 0.8^{m}$ & $56.5^{d}$ & \\
\hline$\rightarrow{ }^{4} \mathrm{D}_{3 / 2}$ & 304.525 & E2: $2.28(-3)$ & $58 \pm 10^{n}$ & & \\
\hline$\rightarrow{ }^{4} \mathrm{D}_{5 / 2}$ & 293.221 & M1: 0.479 & & & \\
\hline & & $\mathrm{E} 2: 4.32(-3)$ & & & \\
\hline$\rightarrow{ }^{4} \mathrm{D}_{7 / 2}$ & 293.221 & M1: 7.30 & & & \\
\hline & & $\mathrm{E} 2: 3.22(-2)$ & & & \\
\hline$\rightarrow{ }^{2} \mathrm{P}_{3 / 2}^{\mathrm{o}}$ & 23.645 & M2: 2.86 & & & \\
\hline & & E3: $4.56(-2)$ & & & \\
\hline$\rightarrow{ }^{2} \mathrm{P}_{1 / 2}^{\mathrm{o}}$ & 24.556 & E3: $3.79(-4)$ & & & \\
\hline
\end{tabular}

\begin{tabular}{|c|c|c|c|}
\hline${ }^{2} \mathrm{G}_{9 / 2} \rightarrow{ }^{4} \mathrm{P}_{5 / 2}$ & 1542.733 & $\mathrm{E} 2: 3.20(-4)$ & $17.8 \pm 3.1^{k}$ \\
\hline$\rightarrow{ }^{2} \mathrm{~F}_{5 / 2}$ & 1142.988 & E2: $9.20(-6)$ & \\
\hline$\rightarrow{ }^{2} \mathrm{~F}_{\tau / 2}$ & 1002.205 & $\begin{array}{c}\mathrm{E} 2: 2.00(-4) \\
\text { M1: } 4.53\end{array}$ & \\
\hline$\rightarrow{ }^{4} \mathrm{~F}_{5 / 2}$ & 415.887 & E2: $8.50(-5)$ & \\
\hline$\rightarrow{ }^{4} \mathrm{~F}_{7 / 2}$ & 357.897 & $\begin{array}{c}\text { E2: } 4.10(-3) \\
\text { M1: } 5.01\end{array}$ & \\
\hline
\end{tabular}




$$
\begin{array}{llc}
\rightarrow{ }^{4} \mathrm{~F}_{9 / 2} & 302.151 & \mathrm{E} 2: 4.49(-2) \\
& & \text { M1: } 51.59 \\
\rightarrow{ }^{4} \mathrm{D}_{5 / 2} & 161.220 & \text { E2: } 3.70(-4) \\
\rightarrow{ }^{4} \mathrm{D}_{7 / 2} & 161.173 & \text { E2: } 1.70(-1) \\
& & \text { M1: } 3.40 \\
\rightarrow{ }^{2} \mathrm{P}_{3 / 2}^{\mathrm{o}} & 22.180 & \text { E3: } 0.757
\end{array}
$$

${ }^{a}$ Extrapolation of heavy-ion storage ring data [54]

${ }^{b}$ Electrostatic ion trap [57]

${ }^{c}$ Electrostatic ion trap [58]

${ }^{d}[65]$

${ }^{e}$ with correction for the electron anomalous magnetic moment (EAMM)

$f$ Extrapolation of heavy-ion storage ring data [56]

${ }^{g}$ Electron beam ion trap [62]

${ }^{h}$ Beam-foil spectroscopy [50]

${ }^{i}[63]$

$j[64]$

${ }^{k}$ Electrostatic ion trap [59]

${ }^{l}$ Heavy-ion storage ring [80]

$m$ Electrostatic ion trap [60]

${ }^{n}$ Heavy-ion storage ring [53] 